\title{
PERFIL SOCIODEMOGRÁFICO DE IDOSOS COM HEPATITE C EM UM MUNICÍPIO DA AMAZÔNIA OCIDENTAL BRASILEIRA
}

Marinete Flores da Silva ${ }^{1}$, Camila Cristina Salazar da Cunha ${ }^{1}$, Maria Celeste da Costa Diniz ${ }^{1}$, Vandson Arantes Sampaio ${ }^{1}$, Eder Ferreira de Arruda ${ }^{2}$.

1. Graduandos em Biomedicina do Centro Universitário UNINORTE, Rio Branco-AC, Brasil. E-mail: marineteflores@yahoo.com.br.

2. Docente do Centro Universitário UNINORTE, Rio Branco-AC, Brasil.

Recebido em: 15/05/2020 - Aprovado em: 15/06/2020 - Publicado em: 30/06/2020 DOI: 10.18677/EnciBio_2020B39

\section{RESUMO}

A hepatite $C$ é uma doença viral grave que pode progredir para neoplasia de fígado e óbito, estando os idosos com as maiores probabilidades de complicações. Por isso, se objetivou descrever o perfil sociodemográfico dos idosos diagnosticados com Hepatite C no município de em Rio Branco-AC durante o ano de 2018. Para tanto, realizou-se de um estudo observacional descritivo, de corte transversal, com abordagem quantitativa, sendo incluídos todos os 38 casos de Hepatite C confirmados entre indivíduos com idade igual ou superior a 60 anos no ano de 2018. As características sociodemográficas foram coletadas do Sistema de Informação de Agravos de Notificação (SINAN-NET). Observou-se que a prevalência geral de Hepatite $C$ foi de $0,13 \%$, sendo de $0,17 \%$ no sexo masculino e de $0,10 \%$ no feminino. A maior parte dos casos de Hepatite C ocorreu em homens $(60,53 \%)$, de cor parda $(84,21 \%)$ e com o ensino fundamental $(50,0 \%)$ ou não alfabetizado $(18,42 \%)$. Dessa forma, a prevalência de Hepatite C, tanto geral quanto por sexo, foi baixa. Entretanto, configura-se como um problema de saúde na população idosa do município. Portanto, são necessárias medidas preventivas e assistenciais voltadas para a doença.

PALAVRAS-CHAVE: Hepatite C. Infecção. Idoso.

\section{SOCIODEMOGRAPHIC PROFILE OF ELDERLY WITH HEPATITIS IN A WESTERN BRAZILIAN AMAZONIAN MUNICIPALITY}

\section{ABSTRACT}

Hepatitis $C$ is a serious viral disease that can progress to liver cancer and death, with the elderly being more likely to have complications. Therefore, the objective was to describe the sociodemographic profile of the elderly diagnosed with Hepatitis $\mathrm{C}$ in the city of Rio Branco-Ac during 2018. For this purpose, a descriptive observational, cross-sectional study was carried out, with a quantitative approach, including all 38 cases of Hepatitis C confirmed among individuals aged 60 years or over in 2018. Sociodemographic characteristics were collected of the Notifiable Diseases Information System (SINAN-NET). It was observed that the general prevalence of Hepatitis C was $0.13 \%$, being $0.17 \%$ in males and $0.10 \%$ in females. Most cases of Hepatitis C occurred in men $(60.53 \%)$, of color $(84.21 \%)$ and with elementary education $(50.0 \%)$ or illiterate $(18.42 \%)$. Thus, the prevalence of Hepatitis C, both general and by sex, was low. However, it is configured as a health problem in the elderly population of the municipality. Therefore, preventive and care measures aimed at the disease are necessary.

KEYWORDS: Hepatiti C. Infection. Elderly 


\section{INTRODUÇÃO}

A hepatite consiste em uma inflamação das células do fígado, podendo ser causada por vírus, uso de drogas lícitas e ilícitas, pela ação de medicamentos hepatotóxicos, assim como em decorrência de doenças genéticas, autoimunes e metabólicas (SOARES et al., 2015). No que se refere às hepatites virais, atualmente, são conhecidas as do tipo A, B, C, D, E, G e TT. No entanto, a Hepatite C se destaca por ser a responsável pela maior parte dos óbitos por hepatites virais no Brasil e representa a terceira maior causa de transplantes hepáticos (BRASIL, 2019a).

A Hepatite $\mathrm{C}$ tem como agente etiológico o vírus $\mathrm{VHC}$ que pertence ao gênero Hepacivirus e família Flaviviridae, tendo seu genoma constituído por uma fita simples de RNA (ICTV, 2020). Os principais fatores de risco para a infecção são: transfusão de hemoderivados de doadores não rastreados com anti-VHC, compartilhamento de material para uso de drogas intravenosas, transplante de órgãos, hemodiálise, uso coletivo de material de higiene pessoal, transmissão vertical, exposição sexual e ocupacional e ausência de vacina ou profilaxia eficaz após exposição ao vírus (YEN et al., 2003; MARTINS et al., 2011; BRASIL, 2019a).

Dependendo da carga viral e das condições clínicas gerais do indivíduo, as consequências mais comuns da infecção pelo VHC são: casos assintomáticos, hepatite aguda ou crônica, cirrose, neoplasia de fígado e óbito (GAZE et al., 2013).

Estima-se que 171 milhões de pessoas estejam infectadas pelo VHC no mundo, o que corresponde a $1 \%$ da população mundial com a forma crônica da doença (WHO, 2017). No Brasil, entre os anos 1999 e 2018, foram confirmados 632.814 casos de hepatites virais. No Acre foi registrado um total de 107 casos de Hepatite C no ano 2018, registrando uma incidência de 18,2 casos por 100 mil habitantes, este valor está acima do índice nacional que é de 12,6 casos por 100 mil habitantes (BRASIL, 2019b).

O VHC pode infectar todos os sexos, raças, todas as faixas etárias, porém, nas últimas décadas têm se evidenciado um aumento dos casos da doença entre os idosos (ALVES et al., 2017). No Brasil, entre os anos de 2008 e 2014, foram registrados 11.567 de óbitos por hepatite $C$ entre idosos (PERAZZO et al., 2017).

Neste contexto, a Hepatite $\mathrm{C}$ é importante devido às graves consequências que acarreta, principalmente entre os idosos. Deste modo, se torna necessário conhecer e caracterizar o perfil dos idosos acometidos, no intuito de estabelecer medidas e ações de prevenção e controle do VHC. Dessa forma, o objetivo deste estudo foi descrever o perfil sociodemográfico dos idosos diagnosticados com Hepatite C no município de Rio Branco-AC durante o ano de 2018.

\section{MATERIAIS E MÉTODOS}

Tratou-se de um estudo observacional descritivo, de corte transversal, com abordagem quantitativa sobre os casos de Hepatite $\mathrm{C}$ entre idosos no município de Rio Branco-AC no ano de 2018.

O município de Rio Branco, capital do estado do Acre, está localizado na Amazônia Sul Ocidental (latitude: 958'26"S; longitude: 6748'27'O), possui uma área territorial de $8.834,942 \mathrm{~km}^{2}$ e população estimada em 407.319 habitantes no ano de 2019 (IBGE, 2020).

Os dados foram obtidos do Sistema de Informação de Agravos de Notificação (SINAN-NET) no mês de março de 2020, sendo incluídos todos os casos de Hepatite C notificados e confirmados entre indivíduos com idade igual ou superior a 
60 anos, destes foram coletadas informações sobre as características sociodemográficas: sexo, raça, faixa etária e escolaridade.

Para a classificação e elegibilidade, foram considerados como casos de Hepatite $\mathrm{C}$, todos os registros que utilizaram os códigos B17.1 a B18.1 presentes no Capítulo I (doenças infecciosas e parasitárias) da Décima Revisão da Classificação Internacional de Doenças e Problemas Relacionados à Saúde (CID-10).

Por meio do programa Microsoft ${ }^{\circledR}$ Office Excel 2016 foram calculadas a prevalência e as frequências absolutas e relativas das características dos indivíduos. A prevalência foi definida como a razão entre o número total de casos de Hepatite $C$ entre os idosos e o número total da população de idosos estimada pelo Instituto Brasileiro de Geografia e Estatística (IBGE) para o ano de 2018 no município de Rio Branco-AC, expressa em porcentagem.

\section{RESULTADOS E DISCUSSÃO}

No município de Rio Branco-AC, no ano 2018, foram notificados 38 casos de Hepatite C. Neste mesmo período, a prevalência geral da doença nos idosos foi de $0,13 \%$. Porém, quando estratificada por sexo, a prevalência nos homens e mulheres foi de $0,17 \%$ e $0,10 \%$, respectivamente (Tabela 1 ).

No estudo realizado por Alves et al. (2017), no estado da Paraíba sobre hepatites virais no período de 2011 a 2015 foi encontrado que 80,1\% dos casos de hepatite em idosos causados pelos vírus B e C. Por sua vez, Silva et al. (2018) ao analisarem os casos de hepatites virais entre idosos do Rio Grande do Norte verificaram que o maior número de notificações foi do vírus tipo C. Já Bonfim et al. (2020) constataram em suas pesquisas um aumento de $52,8 \%$ de indivíduos com idade superior a 60 anos com hepatite C no período de 2016 a 2019 no Piauí.

A baixa prevalência de Hepatite $C$ verificada entre os idosos neste estudo pode ser atribuída às limitações existentes para a investigação dos casos e notificação da doença ao SINAN, principalmente, em virtude de falhas na cobertura e assistência em saúde, problemas na qualidade de informações e dificuldades de diagnóstico, sobretudo quando a infecção progride de forma assintomática (SILVA et al., 2018).

TABELA 1. Prevalência de Hepatite $C$ entre idosos do munícipio de Rio Branco, Acre, Brasil, 2018.

\begin{tabular}{cccc}
\hline \multirow{2}{*}{ No de casos notificados* $^{*}$} & Prevalência geral (\%) & \multicolumn{2}{c}{$\begin{array}{c}\text { Prevalência por gênero } \\
(\%)^{\star *}\end{array}$} \\
\cline { 3 - 4 } & & \multicolumn{2}{c}{$\begin{array}{c}\text { Masculino } \\
\text { Feminino }\end{array}$} \\
\hline 38 & 0,13 & 0,17 & 0,10 \\
\hline
\end{tabular}

Fontes: (SINAN-NET, 2020; IBGE, 2020).

Nota: ${ }^{* *}$ População de idosos: Total=28.177; Masculina=13.152; Feminina=15.025.

Com relação ao sexo, a maior parte dos casos de Hepatite $\mathrm{C}$ ocorreu entre idosos do sexo masculino $(60,53 \%)$, conforme tabela 2 . Este achado corrobora com os resultados de outro estudo desenvolvido no próprio município de Rio Branco-AC, no período de 2001 a 2013 , no qual $63,9 \%$ dos casos de Hepatite $C$ eram homens (FUJIMOTO et al., 2019). De igual modo, em Sergipe, no período de 2007 a 2015, $62,1 \%$ de indivíduos infectados por VHC era do sexo masculino (SANTOS et al., 2017).

Os homens apresentam fatores comportamentais de risco que predispõem a infecção pelo VHC e outras infecções sexualmente transmissíveis (IST), 
principalmente, elevado número de parceiros sexuais e resistência ao uso de preservativos (FRUGOLI; MAGALHÃES-JUNIOR, 2011; FERREIRA et al., 2019).

Farias et al. (2019), ao elaborarem um mapa das hepatites virais, no período de 2010 a 2014, verificaram que o município de Rio Branco apresentou a maior taxa de frequência da hepatite $C$ do estado do Acre (30,33\%), sendo os homens os mais afetados $(62,60 \%)$, sobretudo através do contato sexual $(66,0 \%)$. Da mesma forma, Amaral et al., (2013) verificaram que $65,0 \%$ dos casos de Hepatite $\mathrm{C}$ em Rio Branco eram do sexo masculino, principalmente acima dos 40 anos.

Vieira et al. (2014) ao estudarem a ocorrência e distribuição espacial da hepatite C no estado de Rondônia durante o período 2002 a 2012 verificaram que $63,1 \%$ casos eram do sexo masculino. De maneira similar, uma pesquisa em Minas Gerais sobre o panorama etiológico do vírus da hepatite $\mathrm{C}$ verificou maior frequência de infecção no sexo masculino com 54,0\% (BANDEIRA et al., 2018).

TABELA 2 - Características sociodemográficas de idosos no município de Rio Branco, Acre, 2018.

\begin{tabular}{lcc}
\hline Variável & $\mathbf{N}$ & $\%$ \\
\hline Sexo & & \\
Masculino & 23 & 60,5 \\
Feminino & 15 & 39,5 \\
Raça & & \\
Branca & 04 & 10,5 \\
Parda & 32 & 84,2 \\
Não informado & 02 & 5,3 \\
Escolaridade & & \\
Não alfabetizado & 07 & 18,4 \\
Ensino fundamental & 19 & 50,0 \\
Ensino médio & 02 & 5,3 \\
Ensino superior & 04 & 10,5 \\
Não informado & 06 & 15,8 \\
\hline Total & 38 & $\mathbf{1 0 0 , 0}$ \\
\hline
\end{tabular}

Fonte: SINAN-NET, 2020.

Concernente à cor ou raça, $84,21 \%$ dos idosos eram pardos (Tabela 2). Resultado similar foi obtido por Santos et al. (2017) em Sergipe no período de 2007 a 2015 que verificaram que a maior taxa de hepatite $C$ ocorreu em indivíduos da cor parda $(66,1 \%)$. A análise da cor ou raça relacionada com casos de hepatite $\mathrm{C}$ revelou que a raça parda foi a mais prevalente em uma pesquisa realizada em Minas Gerais no ano de 2018 com 38,0\% (BANDEIRA et al., 2018). Do mesmo modo, no Pará, no período de 2010 a 2014 a raça parda também prevaleceu apresentando a maior taxa de frequência de hepatite C com 65,1\% (GONÇALVES et al., 2019).

Essa predominância de casos de hepatite $C$ da raça parda pode ser explicada devido ao fato de que mais da metade da população brasileira, principalmente nas regiões Norte e Nordeste, se autodeclara como da raça parda ou misto (OLIVEIRA; LUIZ, 2019).

No que se refere à escolaridade, houve predomínio de baixo nível de instrução no qual 18,42\% não eram alfabetizados e 50,0\% tinham o ensino fundamental (Tabela 2). Dados semelhantes também foram identificados por Ferreira et al. (2019) ao estudarem a vulnerabilidade de idosos a IST, 68,0\% dos 
idosos tinham apenas o ensino fundamental e 20,17\% não tinham nenhum grau de instrução.

Amaral et al. (2013) ao investigarem as características socioeconômicas e demográficas dos portadores de hepatite C em Rio Branco, no período de 2006 a 2007 identificaram que 45,9\% dos portadores de hepatite C, apresentavam apenas ensino fundamental. Outras pesquisas também encontraram predominantemente, participantes com ensino fundamental (MOIA et al., 2014; GONÇALVES et al., 2019).

A baixa escolaridade dos idosos é um fator que contribui para dificuldades de compreensão de informações acerca da Hepatite C e outras IST. Assim tornando-os mais vulneráveis e suscetíveis devido ao baixo nível de conhecimentos e a menor autonomia na adoção e aceitação de medidas preventivas e de autocuidado em saúde (SILVA et al., 2011; FERREIRA et al., 2019).

\section{CONCLUSÕES}

Embora, a prevalência de Hepatite $C$ entre os idosos do município de Rio Branco-AC não tenha sido elevada, mas a infeção está presente na população e configura-se como um relevante problema de saúde pública.

Portanto, verifica-se a necessidade do estabelecimento de medidas e ações de saúde que priorizem a prevenção, diagnóstico, acompanhamento e tratamento da doença entre os idosos. Bem como, também, o desenvolvimento de novos estudos voltados para esta faixa etária, o que consequentemente favorecerá a um possível mapeamento da doença e pode subsidiar melhorias nas estratégias de prevenção e na assistência prestada aos idosos.

\section{REFERÊNCIAS}

ALVES, B. P.; SANTANA, J. D. L.; NASCIMENTO, J. G. C.; NUNES, P. T. C. M.; FERREIRA, S. B. Comportamento epidemiológico da infecção pelos vírus da hepatite B e C em idosos no estado da Paraíba. In: V Congresso Internacional de Envelhecimento Humano, 22-24 de novembro, Maceió, Alagoas, 2017, 10 p. Disponível em: <https://editorarealize.com.br/revistas/cieh/trabalhos/TRABALHO_EV075 MD2 SA9 ID741_29082017231158.pdf>.

AMARAL, T. L. M.; RODRIGUES, A. U.; QUEIROZ, M. M. C. Perfil clínico e epidemiológico da hepatite $C$ em Rio Branco, Acre, Brasil. Revista de Saúde, v.9, n.2, p.64-79, 2013. Disponível em: <https://www.arca.fiocruz.br/handle/icict/11045>.

BRASIL. Protocolo clínico e diretrizes terapêuticas para hepatite c e coinfecções. Brasília: Ministério da Saúde, 2019a. 143 p. Disponível em: $<$ http://www.aids.gov.br/pt-br/pub/2017/protocolo-clinico-e-diretrizes-terapeuticaspara-hepatite-c-e-coinfeccoes $>$.

BRASIL. Boletim Epidemiológico Hepatites Virais. Brasília: Ministério da Saúde, 2019b. 76 p. Disponível em: <http://www.aids.gov.br/pt-br/pub/2019/boletimepidemiologico-de-hepatites-virais-2019>.

BONFIM, K. L. F.; NASCIMENTO JUNIOR, W.; NASCIMENTO, W. L.; JOSÉ AYRTON DE SOUZA COSTA, J. A. S. C.; SOUZA, R. P. et al. Perfil clinico epidemiológico de portadores de hepatite $c$ do estado do Piaui. Brazilian Journal of 
Surgery and Clinical Research, v.30, n.2, p.06-10, 2020. Disponível em: $<$ http://www.mastereditora.com.br/bjscr>.

BANDEIRA, L. L. B.; SOUZA, C. S.; PERUZINI, G. A.; MARQUES, D. R.; GUEDES, L. V. et al. Epidemiologia das hepatites virais por classificação etiológica. Revista da Sociedade Brasileira e Clínica Médica, v.16, n.4, p.227-231, 2018. Disponível em: <http://www.sbcm.org.br/ojs3/index.php/rsbcm/article/view/376>.

FRUGOLI, A.; MAGALHÃES-JUNIOR, C. A. O. A sexualidade na terceira idade na percepção de um grupo de idosas e indicações para a educação sexual. Arquivo de Ciência da Saúde, v. 15, n.1, p.85-93, 2011. Disponível em: <https://doi.org/10.25110/arqsaude.v15i1.2011.3696>

FERREIRA, C. O.; DAVOGLIO, R. S.; VIANNA, A. S. A.; SILVA, A. A.; REZENDE, E. A.R. et al. Vulnerabilidade a infecções sexualmente transmissíveis em idosos usuários de um centro de testagem e aconselhamento. Arquivo de Ciências da Saúde, v. 23, n.3, p.171-180, 2019. Disponível em: <https://doi.org/10.25110/arqsaude.v23i3.2019.6757>.

FARIAS, C. S.; OLIVEIRA, R. A. D.; LUZ, M. R. M. P. O mapa das hepatites virais no Acre: entre territórios e territorialidades. Revista Brasileira de Geografia Física, v.12, n.6 p.2339-2354, 2019. Disponível em: <10.26848/rbgf.v12.6.p2339-2354>

FUJIMOTO, D. E.; WARDEN, C. F.; KOIFMAN, R. J. Tendência temporal da incidência de hepatite viral $B$ e $C$ no Estado do Acre, no período de 2001 a 2013. Dê Ciência em Foco, v.3, n.2, p.81-96, 2019. Disponível em: <http://revistas.uninorteac.com.br/index.php/DeCienciaemFoco0/article/view/322>.

GAZE, R.; CARVALHO, D. M.; LOPES, G. S.; TURA, L. F. R. Das hepatopatias e icterícias às hepatites virais: configuração de um caleidoscópio. Revista de Saúde Pública, v. 47, n.1, p.116-122, 2013. Disponível em: $<$ https://www.scielo.br/pdf/rsp/v47n1/15.pdf>.

GONÇALVES, N. V.; VIEIRA, D. C.; MIRANDA, C. S. C.; PALÁCIOS, R. C. M.; COSTA, S. B. N. et al. Análise espacial e epidemiológica de hepatites B e $C$ e índice de desenvolvimento humano municipal, no estado do Pará. Hygeia, v. 15, n.31, p.29-42, 2019. Disponível em: <10.1590/1414-462X201900010394>.

ICTV - International Committee on Taxonomy of Viruses . Reports on Vírus Taxonomy. 2020. Disponível em: <https://talk.ictvonline.org>.

IBGE - Instituto Brasileiro de Geografia e Estatística. Rio Branco (AC). 2020. Disponível em: <https://cidades.ibge.gov.br/brasil/ac/rio-branco/panorama>.

MARTINS, T.; SCHIAVON, J. L. N.; SCHIAVON, L. L. Epidemiologia da infecção pelo vírus da hepatite C. Revista da Associação Medica Brasileira, v.57, n.1, p.107-112, $2011 . \quad$ Disponível em <https://www.scielo.br/pdf/ramb/v57n1/v57n1a24.pdf>. 
MOIA, L. J. M. P.; AMARAL, I. S. A.; FARIAS, A. J. L.; SILVA, M. M. Infecção pelo vírus da hepatite $C$ em hospital de referência na amazônia brasileira. Revista Paraense de Medicina, v.28, n.3, p.19-25, 2014. Disponível em: <https://files.bvs.br/upload/s/0101-5907/2014/v28n3/a4511.pdf>

OLIVEIRA, B. L. C. A.; LUIZ, R. R. Densidade racial e a situação socioeconômica, demográfica e de saúde nas cidades brasileiras em 2000 e 2010. Revista Brasileira de Epidemiologia, v. 22, p. 1-12, 2019. Disponível em: <10.1590/1980549720190036>

PERAZZO, H.; PACHECO, A. G.; LUZ, P. M.; CASTRO, R.; HYDE, C. et al. Agestandardized mortality rates related to viral hepatitis in Brazil. BioMedCentral Infectious Diseases, v. 17, n.527, p.1-10, 2017. Disponível em: <https://doi.org/10.1186/s12879-017-2619-y>.

SOARES, C. C.; NIEL, C. M. G.; MELLO, F. C. A.; GOMES, S. A. Hepatites Virais In: SANTOS, N. S.O.; ROMANOS, M. T. V.; WIGG, M. S. Virologia Humana, 3. ed. Rio de Janeiro: Guanabara Koogan, 2015, 598p.

SILVA, J. F. C.; MORAIS, N. S.; SILVA, D. R.; SANTOS, J. S. F.; MARQUES, E. M. Hepatites virais na terceira idade: casos do Rio Grande do Norte, Brasil. Brazilian Journal of health Review, v.1, n.1, p.19-27, 2018. Disponível em: $<$ http://www.brjd.com.br/index.php/BJHR/article/view/547>.

SANTOS, A. D. S.; ARAÚJO, D. C.; LIMA, S. V. M. A.; GÓES, M. A. O.; MENEZES, A. F. et al. Dinâmica espacial e temporal da infecção pelo vírus da hepatite $C$. Arquivos de Ciência da Saúde, v.24, n.4, p.14-19, 2017. Disponível em: <doi.org/10.17696/2318-3691.24.4.2017.802>.

SILVA, H. R.; MARREIROS, M. Ó. C.; FIGUEIREDO, T.S.; FIGUEIREDO, M. L. F. Características clínico-epidemiológicas de pacientes idosos com aids em hospital de referência, Teresina-PI, 1996 a 2009. Epidemiologia no Serviço de Saúde, v. 20, n.4, p.499-507, 2011. Disponível em: <http://dx.doi.org/10.5123/S167949742011000400009>.

VIEIRA, G.D.; VENTURA, C. G.; SOUSA, C. M. Occurrence and spatial distribution of Hepatitis $C$ in a western brasilian amazon state. Arquivo de Gastroenterologia, v.51, n.4, p.316-319, 2014. Disponível em: <https://www.scielo.br/pdf/ag/v51n4/00042803-ag-51-04-316.pdf>.

WHO. WORLD HEALTH ORGANIZATION (WHO). Global Hepatitis Report, Genebra, 2017, 83p. Disponível em: <https://apps.who.int/iris/bitstream/handle/10665/255016/9789241565455eng.pdf;jsessionid=B0A6524A7A0CDC326A4F75C66D0FAFFF? sequence=1 $>$.

YEN, T.; KEEFFE, E. B.; AHMED, A. The epidemiology of hepatitis c virus infection. Journal of Clinical Gastroenterology, v.36, n. 1, p.47-53, 2003. Disponível em: <https://journals.Iww.com/jcge/Pages/ArticleViewer.aspx?year=2003\&issue =01000\& article $=00015 \&$ type $=$ Fulltext $>$. 